Mo S2b 05

\title{
Management of Sinkhole Risks Using Long Term ERT Monitoring - A Laboratory Experiment
}

\author{
J. Deceuster* (University of Mons), J.E. Chambers (British Geological \\ Survey), P. Goderniaux (University of Mons), O. Kuras (British Geological \\ Survey), P.B. Wilkinson (British Geological Survey) \& O. Kaufmann \\ (University of Mons)
}

\section{SUMMARY}

Accurate methodologies are required to manage risks linked to land-use planning in covered kart terrains, especially in densely urbanized areas. The main risk lies in the occurrence of sinkholes at the base of buildings or infrastructure. We conducted a laboratory experiment to evaluate the contribution of ERT monitoring in the long term management of such karstic risks. After presenting the design of the laboratory experiment, we detail the selected scenarios and the acquisition protocols tested. The methodology proposed to process the data and manage the inversion results relies on two steps: (1) we estimate the resistivity variations due to measurement and inversion errors based on Monte-Carlo simulations and (2) we define a resistivity changes index for every cell of the 3D model. The methodology is tested on a 3D surface survey including inline dipole-dipole, equatorial dipole and Wenner-Schlumberger arrays. A $10 \mathrm{~cm}$ in diameter plastic ball is used to model a $3.5 \mathrm{~m}$ sinkhole at depths ranging from the surface to $20 \mathrm{~cm}$, using a water resistivity of $12 \mathrm{Ohm} . \mathrm{m}$. Based on the proposed methodology, we show that this target can be suitably detected when its top reaches $10 \mathrm{~cm}$ or less in depth. 


\section{Introduction}

When considering the world's demographic growth in relation to international land planning programs that dedicate more and more zones to non-building purposes, like agriculture or nature preservation, it is obvious that the supply is restrained while the demand is expanding. This context leads more and more contemporary builders to settle industrial or residential projects in zones that remain "available for erecting usages", whatever the suitability of ground capabilities or the compatibility with the surrounding environment. When erecting buildings or infrastructure in covered karst terrains, builders and owners should take into account hazards linked with sinkhole activity. Frequently, direct investigations (e.g., core sampling, cone penetration tests, digging) or non-invasive geophysical surveys are carried out to estimate the hazard level at the local scale prior to the construction of the project. When dealing with existing structures or when the karstic hazard level is moderate to high or cannot be estimated due to specific site conditions (e.g., available space at the surface, targets depth), long term monitoring techniques are needed to manage risks during the life of the considered project.

For at least two decades, electrical resistivity tomography (ERT) experiments have been widely conducted to detect and map karstic features such as weathered zones (e.g., Kaufmann et al., 2012) and voids or cavities sometimes partly or completely filled with water or allochthonous sediments (e.g., Valois et al., 2011). Over the last few years, long-term ERT monitoring experiments have been conducted to assess the potential of the method in managing natural hazards, such as landslides or frozen grounds, and engineering and environmental problems, such as dams, landfills, leak detection and tracer tests. This on-going study aims to assess the potential and limitations of the ERT monitoring in detecting sinkholes before they reach the surface considering different practical scenarios modelled through a laboratory experiment.

\section{Design of the laboratory experiment}

The test tank designed to model the selected scenarios in laboratory consists of a $1 \mathrm{~m}^{3}$ PVC tank (Figure 1A). Commonly, in covered karst terrains, a cover thickness of at least 10 meters should be expected. In Belgium, the resistivity of the cover materials ranges from a few tens of Ohm.m to more than $1000 \mathrm{Ohm} . \mathrm{m}$. To simulate these conditions, the tank was first filled with demineralized water $(\sim 1.8 \mu \mathrm{S} / \mathrm{cm} \equiv 5550 \mathrm{Ohm} . \mathrm{m})$. The water resistivity is checked during the monitoring experiment using a conductivity probe. $\mathrm{NaCl}$ is added to the water to gain lower resistivities when needed. To prevent a resistivity gradient linked with salt sedimentation when higher background conductivities are modelled, an isostatic pump is used to mix the fluid from bottom to top. The tank is also equipped with a temperature probe to account for resistivity variations due to temperature drift.

Figure 1C shows the electrode arrangement considered for this study. It consists of 48 surface electrodes located on a grid of $6 \times 8$ and 8 boreholes of 6 electrodes placed at selected locations. Electrodes are spaced $14 \mathrm{~cm}$ from each other, both at the surface and in boreholes. Surface electrodes are made of stainless steel bolts (grade 316L), $5 \mathrm{~mm}$ in diameter. Electrodes in boreholes consist in stainless steel wires (grade $316 \mathrm{~L}$ ), $0.5 \mathrm{~mm}$ in diameter. The positioning system was designed to minimize electrode positioning errors, to ensure that surface electrodes are immersed of a constant depth and to prevent unexpected movements of the electrodes during data acquisition (Figure 1B).With such an electrodes arrangement, surface quadrupole, cross-borehole and surface-to-hole measurements can be tested.

Sinkholes are modelled using plastic balls of different sizes. Apparent resistivity measurements are conducted using a Syscal Pro resistivity meter (Iris Instruments, France). The intensity of the current pulse is fixed at $12 \mathrm{~V}$ to limit the current density flowing in the water to values similar to those observed in the field. The electrodes were connected to the resistivity meter through a switching box using shielded cables to prevent cross talk.

\section{Selected scenarios}

According to our knowledge of the geological background, we selected three resistivity values typically encountered in cover materials found in karstic areas in Belgium: 10 Ohm.m (clays), 55 
Ohm.m (silts, marls and chalks) and 555 Ohm.m (sands). Expected sinkholes diameters should range between 3 and 15 meters when occurring at the surface. At the model scale, the corresponding target sizes range from 8 to $40 \mathrm{~cm}$ in diameter. The target depths range from the surface to $90 \mathrm{~cm}$, corresponding to a $30 \mathrm{~m}$ depth on the field. To assess the detection limitations, the plastic balls are displaced by $7 \mathrm{~cm}$ to reach the bottom of the tank. Initially, the targets are located at the centre of the tank.

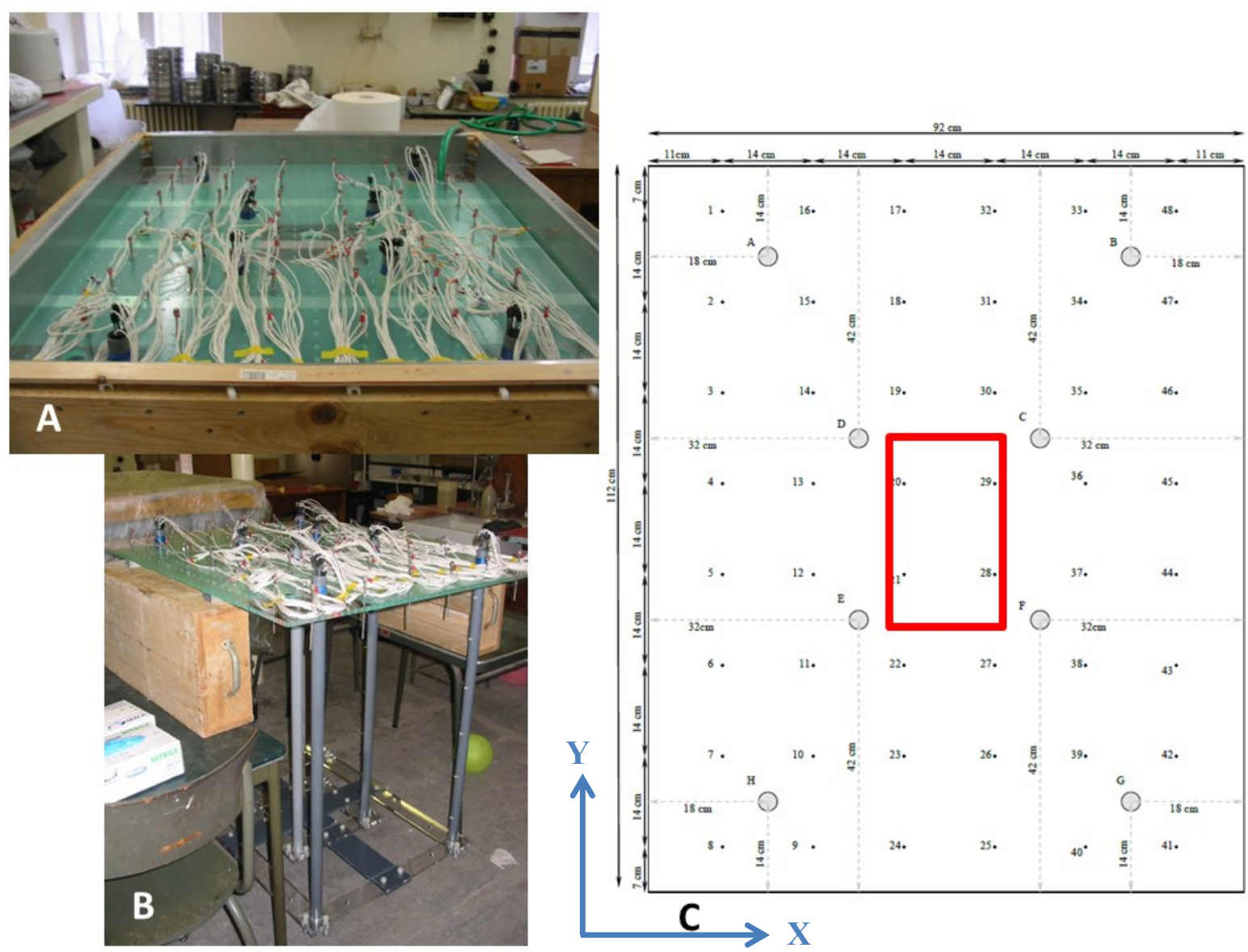

Figure $1 A \& B$ : Photographs of the test tank with surface and borehole electrodes. C: Sketch of the electrodes arrangement used for the ERT monitoring experiment. The simulated typical footprint of a family home is delineated in red.

In this study, we consider the management of karstic risks on both building projects (characterized by lands free from occupation) and existing buildings. When dealing with construction projects, we assume that a 3D grid of electrodes can be arranged before the start of the monitoring as well as electrodes in boreholes. When the building is still present, three cases are studied: (1) cross-hole measurements alone, (2) loop arrays (electrodes are located around the building) and (3) surface-tohole measurements.

\section{Survey design}

As a first step, several commonly used electrode array configurations are considered as well as combinations of these array configurations. For the 3D surface acquisition, inline dipole-dipole, Wenner-Schlumberger and equatorial dipole arrays are used. For the cross-hole measurements, only the AM-BN dipole array is tested. For surveys with loop arrays, common inline array configurations and cross-lines array configurations are investigated. When considering surface-to-hole measurements, arrays with a single current electrode in each borehole are investigated. In a second step, we optimize the 3D surface acquisition protocol by adapting the survey optimization algorithm proposed by Wilkinson et al. (2012). 


\section{Processing the data and managing the inversion results}

The acquired datasets are filtered based on V/I ratios, repeatability errors and reciprocal error measurements. Filtered data are then inverted separately within ERTLab+ software (Geostudy Astier, Italy). We use a robust inversion scheme on both data and model constraints as sharp boundaries in resistivities are expected between targets and water. The tank geometry is taken into account by defining Neumann boundary conditions on the 3D model.

Several background datasets were acquired to evaluate measurements errors when the tank is just filled with water. Assuming normal distributions of these errors, we generated 200 random draws which follow the estimated distributions. We then performed Monte Carlo simulations to estimate the induced errors on the inverted resistivities. As these errors show a normal distribution, we defined resistivity intervals for every cells of the model using the 2 and $3 \sigma$ confidence intervals (Coppens et al., 2012). The inverted models, computed when targets are present, are compared to these 2 and $3 \sigma$ confidence intervals. We then defined a resistivity changes index RCI (equation 1) to identify the significant changes in resistivities. This index may be used as alpha transparencies when displaying percentage change in resistivities (PCR) due to the target.

$R C I=\left\{\begin{array}{c}0, \text { when } \rho(x, y, z) \leq(\mu+2 \sigma)_{x, y, z} \\ \frac{\rho(x, y, z)-(\mu+2 \sigma)_{x, y, z}}{\sigma}, \text { when }(\mu+2 \sigma)_{x, y, z}<\rho(x, y, z)<(\mu+3 \sigma)_{x, y, z} \\ 1, \text { when } \rho(x, y, z) \geq(\mu+3 \sigma)_{x, y, z}\end{array}\right.$

\section{Preliminary results}
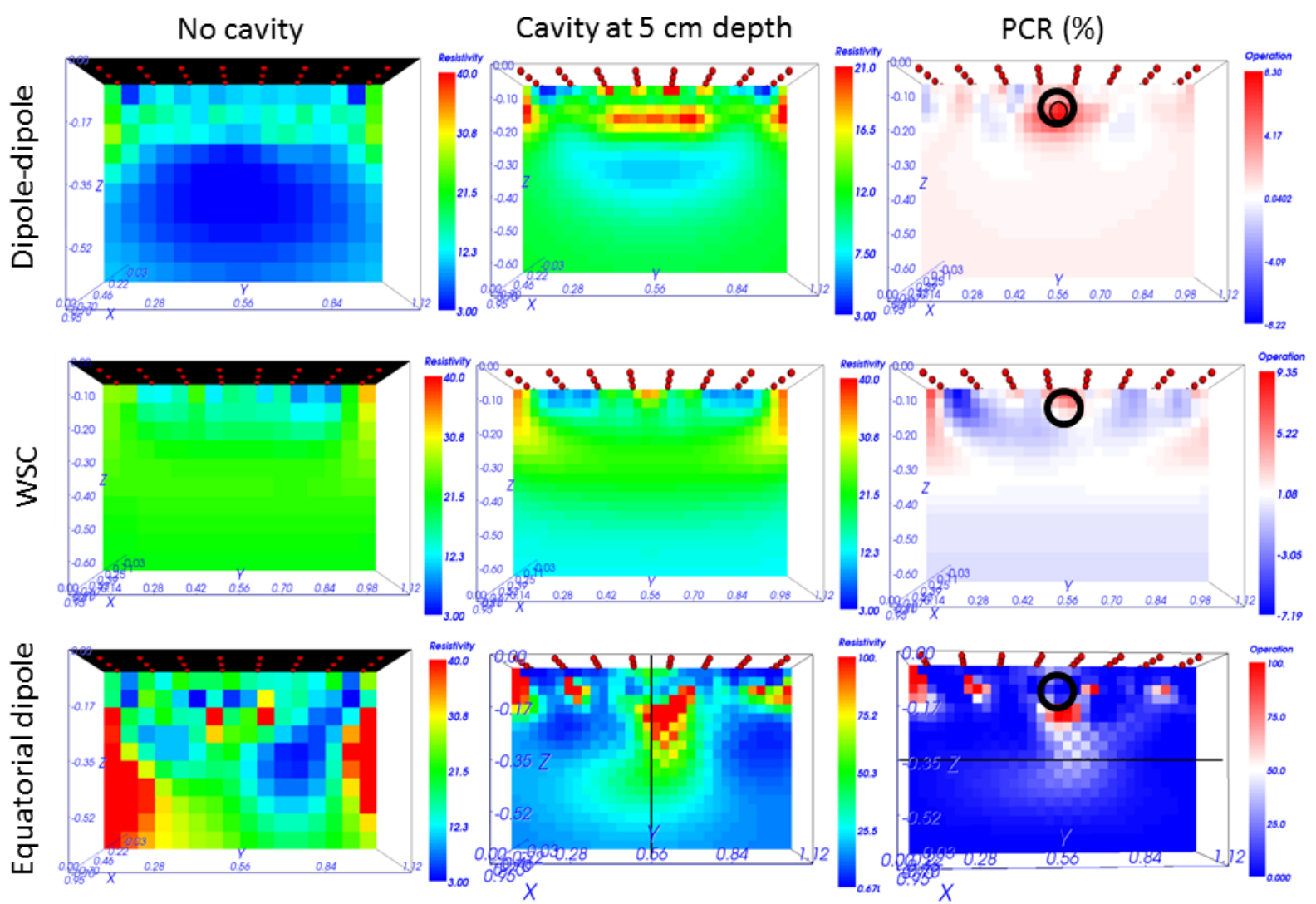

Figure 2 Plots of the resistivity models obtained after the inversion of background datasets (no cavity) and of datasets acquired with a plastic ball $(10 \mathrm{~cm}$ in diameter and top of the target located at $5 \mathrm{~cm}$ in depth). The percentage changes in resistivity (PCR) plots are computed for the selected array configurations. (Water resistivity $=12$ Ohm.m) 
At this stage of the study, we focus on the ERT monitoring of building projects and we consider 3D surface measurements. Figure 2 illustrates some preliminary results of the study for the selected array configurations. The computed resistivity changes indexes (RCI) are plotted on Figure 3 for the $10 \mathrm{~cm}$ cavity located at depths ranging from the surface to $15 \mathrm{~cm}$ (case A to E) and for a cavity located at 5 $\mathrm{cm}$ in depth but located with a $21 \mathrm{~cm}$ offset along the $\mathrm{Y}$ axis.
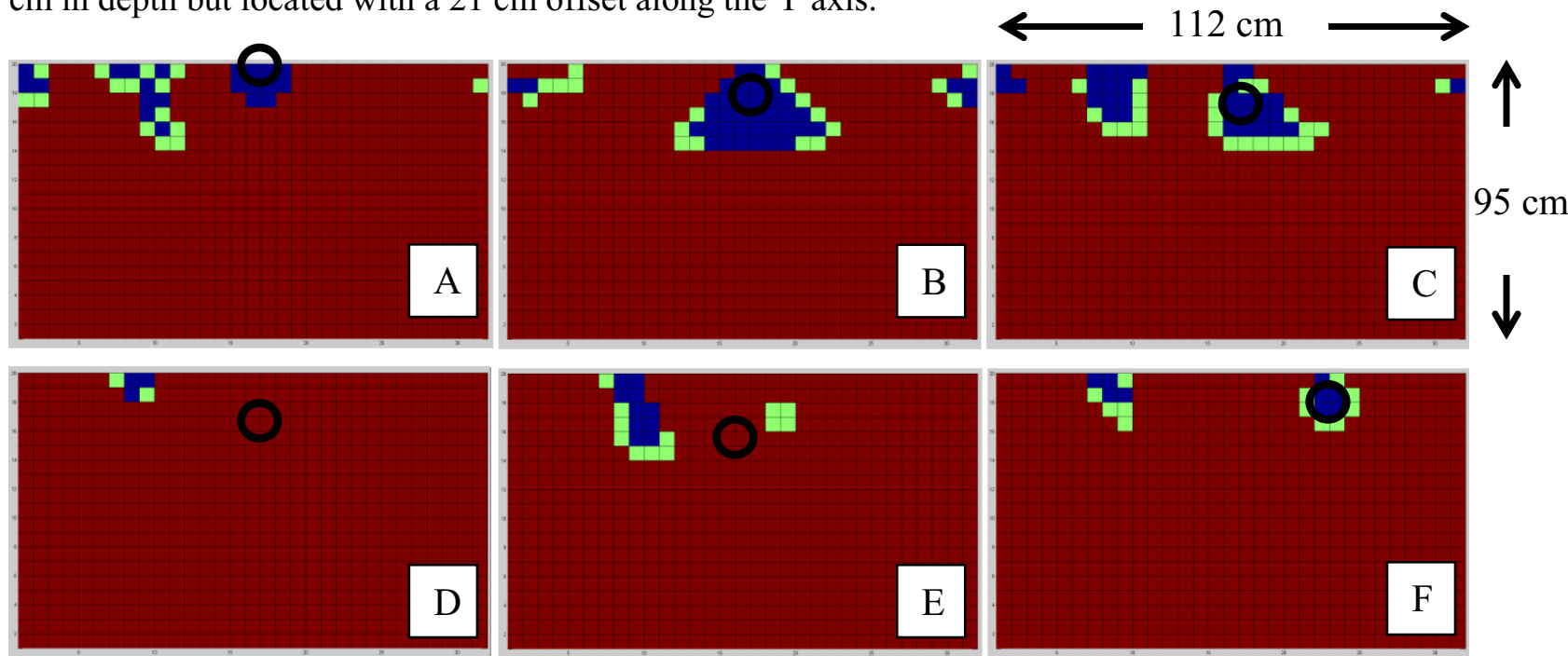

Figure 3 Plots of the RCI values for the dipole-dipole survey with A: cavity at the surface, B: cavity at $5 \mathrm{~cm}$ in depth, C: cavity at $7 \mathrm{~cm}$ in depth, D: cavity at $11 \mathrm{~cm}$ in depth, E: cavity at $15 \mathrm{~cm}$ in depth and F: cavity with a $21 \mathrm{~cm}$ offset at $5 \mathrm{~cm}$ in depth (after Coppens et al., 2012). These sections are extracted from the $3 D$ model at the centre of the tank and are aligned along the $Y$ axis.

\section{Conclusions and perspectives}

The preliminary results showed that this $10 \mathrm{~cm}$ in diameter target can be suitably detected until its top reach $10 \mathrm{~cm}$ in depth. This tends to indicate that the selected methodology is suitable for assessing the efficiency of the ERT monitoring technique in detecting sinkholes occurrences at the local scale. This study is still on-going and future works will focus on the assessment of the limitations and pitfalls of the technique.

\section{Acknowledgments}

This work is granted by the Walloon Region Ministry (FIRST SPIN-OFF GRANSPE visa $\mathrm{n}^{\circ}$ 091/6974) and the Académie Universitaire Wallonie-Bruxelles (Fonds de Maturation GRANSPE). We wish to thank particularly E. Legrand from the University of Mons for his valuable help in the design and realization of the test tank and during the data acquisition.

\section{References}

Coppens J., Dumont R., Laret T., Wuyckens G., 2012. Utilisation de la géophysique pour le monitoring et la gestion des risques karstiques. Rapport du projet en environnement. UMONS, unpublished.

Kaufmann O., Deceuster J., Quinif Y., 2012. An electrical resistivity imaging-based strategy to enable site-scale planning over covered palaeokarst features in the Tournaisis area (Belgium). Engineering Geology 133-134, 49-65.

Valois R., Camerlynck C., Dhemaied A., Guérin R., Hovhannissian G., Plagnes V., Rejiba F., Robain H., 2011. Assessment of doline geometry using geophysics on the Quercy plateau karst (South France). Earth Surface Processes and Landforms 36 (9), 1183-1192.

Wilkinson P.B., Loke M.H., Meldrum P.I., Chambers J.E., Kuras O., Gunn D.A., Ogilvy R.D., 2012. Practical aspects of applied optimized survey design for electrical resistivity tomography. Geophysical Journal International189, 428-440. 